\title{
Equal, but different: Fluctuant biofilm formation and its impact on polymyxin B susceptibility among a clonal spreading of KPC-2-producing Klebsiella pneumoniae isolates
}

\author{
Leandro Reus Rodrigues Perez PhD (1) \\ Universidade Federal do Rio Grande do Sul, Porto Alegre, Brazil
}

To the Editor-Klebsiella pneumoniae often causes severe nosocomial infections against which carbapenem agents are frequently used when isolates produce broad-spectrum $\beta$-lactamase enzymes (ie, extended-spectrum $\beta$-lactamase [ESBL] or ampC type). In the last decade, carbapenem-resistant $K$. pneumoniae has been increasing worldwide, mainly due to K. pneumoniae carbapenemase (KPC) enzymes. Bla $a_{\mathrm{KPC}-2}$ is especially common in Brazil, conferring resistance to almost all available antibiotics in association with high morbidity and mortality rates., ${ }^{1,2}$

Biofilm formation is a well-recognized pathway by which pathogenic bacteria evade adverse conditions (oxygen and nutrient deprivation) as well as bactericidal antimicrobial agents. Matrixenclosed biofilms are formed preferentially on a variety of surfaces inside the body and on invasive devices. ${ }^{3}$

Recently, we evaluated the ability to produce biofilms among Pseudomonas aeruginosa clinical isolates and their impact on antimicrobial susceptibility profiles; there was an alarming impact when overlapping resistance mechanisms (biofilm plus enzymatic production) were present. ${ }^{4}$ These results prompted a study evaluating these overlapping mechanisms because few reports are available regarding biofilm production among KPC-2-producing K. pneumoniae (KPC-2-Kp) isolates. ${ }^{5,6}$

In the present report, we describe for the first time the ability to produce biofilms among KPC-2-Kp clinical isolates from extensive clonal spread in patients in an intensive care unit. This is a substudy of the evaluation of the impact of biofilms on the potential development of antimicrobial resistance. ${ }^{7}$

We analyzed 10 pairs of KPC-2-Kp isolates from rectal swabs and clinical isolates obtained from 10 unique patients as previously described. ${ }^{2}$ These isolates were subjected to microtiter plate assays performed in triplicate, biofilm status characterization, and determination of both polymyxin B minimum inhibitory concentrations (MIC) and minimum biofilm eradication concentrations (MBEC) as described elsewhere. ${ }^{4}$

All KPC-2-Kp isolates were identical by molecular typing, ${ }^{2}$ but most of them had different biofilm profiles (Table 1). Four pairs of KPC-2-Kp had the same biofilm formation status: 3 of these isolates were recovered from blood and 1 was recovered from cerebrospinal fluid. However, for the remaining 6 pairs, an increase in biofilm production was observed in the clinical isolates compared

\footnotetext{
Author for correspondence: Leandro Reus Rodrigues Perez, Email: leandro.reus@gmail. com

Cite this article: Perez LRR. (2019). Equal, but different: Fluctuant biofilm formation and its impact on polymyxin B susceptibility among a clonal spreading of KPC-2-producing Klebsiella pneumoniae isolates. Infection Control \& Hospital Epidemiology, 40: 954-955, https://doi.org/10.1017/ice.2019.106
}

to their colonizing counterparts (Table 1). A worrisome consequence of these results was the association with increasing resistance levels or bacterial tolerance in the face of higher polymyxin B concentrations. Moreover, polymyxin B MBEC results revealed that $90 \%$ of clinical isolates showed a $2-16$-fold increase in resistance in biofilms (Table 1). Similar to our results, Naparstek et al. ${ }^{5}$ found a dramatic increase in polymyxin resistance with $K$. pneumoniae isolates in biofilms. Although K. pneumoniae are not as notorious for biofilm formation as Pseudomonas aeruginosa, we did find that $K$. pneumoniae clinical isolates indeed produce robust biofilms compared with the same bacterial clone previously recovered from surveillance cultures reflecting gut colonization.

Many bacteria grow on surfaces forming biofilms, but high dosages of antimicrobial often cannot clear infectious biofilms. Most of our isolates were recovered from patients with some invasive medical device (eg, bladder catheter, central venous catheter, or cerebrospinal fluid diversion), which is a well-characterized risk factor for both an infection process and biofilm production. ${ }^{8}$

A potential limitation of this study was the lack of information about the genetic lineage of our KPC-2-Kp isolates. Some evidence shows that ST258 are widely disseminated in many Brazilian hospitals ${ }^{9}$ and that this ST258 lineage has a lower biofilm biomass than other lineages. ${ }^{5}$ Little is known about the ability to produce biofilms in sets of the same KPC-2-Kp clonal isolates when recovered from distinct clinical specimens in the same patient.

In conclusion, a KPC-2-Kp predominant clone exhibits exuberant biofilm formation and high polymyxin $B$ resistance levels, as noted with other species. ${ }^{4,6}$ Although our results should be validated by further studies involving other clonal lineages, the ability to produce biofilm may be responsible for maintaining refractory infections due to KPC-2-Kp isolates, with an impact on polymyxin $B$ resistance development.

Author ORCIDs. Leandro Perez, (iD) 0000-0002-6662-6503

Acknowledgments. The author would like to thank Sophia Perez for technical support.

Financial support. No financial support was provided relevant to this article. Conflicts of interest. All authors report no conflicts of interest relevant to this article.

\section{References}

1. Rodrigues Perez LR. Carbapenem-resistant enterobacteriaceae: a major prevalence difference due to the high performance of carbapenemase producers when compared to the nonproducers. Infect Control Hosp Epidemiol 2015;36:1480-1482. 
Table 1. Biofilm Status for KPC-2-Klebsiella pneumoniae Colonizing and Infecting Isolates and Polymyxin B MBEC/MIC Determination for Infecting Isolates

\begin{tabular}{|c|c|c|c|c|}
\hline Patient & Clinical Site & \multicolumn{2}{|c|}{ Biofilm Status } & Polymyxin B MBEC/MIC (Ratio) \\
\hline Patient 1 & Blood & Nonproducing & Nonproducing & $2.0 / 2.0(1)$ \\
\hline Patient 3 & Urine & Weak & Moderate & $8.0 / 2.0(4)$ \\
\hline Patient 4 & Cerebrospinal fluid & Moderate & Moderate & $8.0 / 2.0(4)$ \\
\hline Patient 6 & Blood & Moderate & Strong & $16.0 / 4.0(4)$ \\
\hline Patient 7 & Endotracheal aspirate & Weak & Strong & $128.0 / 8.0(16)$ \\
\hline Patient 8 & Blood & Weak & Weak & $64.0 / 32.0(2)$ \\
\hline Patient 9 & Blood & Weak & Weak & $1.0 / 0.5(2)$ \\
\hline Patient 10 & Urine & Nonproducing & Moderate & $2.0 / 1.0(2)$ \\
\hline
\end{tabular}

Note. MBEC, minimum biofilm eradication concentration; MIC, minimum inhibitory concentration.

2. Rodrigues Perez LR, Dias CG. Emergence of infections due to a polymyxin B-resistant KPC-2-producing Klebsiella pneumoniae in critically ill patients: what is the role of a previous colonization? Infect Control Hosp Epidemiol 2016;37:240-241.

3. Williams DL, Costerton JW. Using biofilms as initial inocula in animal models of biofilm-related infections. J Biomed Mater Res B Appl Biomater 2012;100:1163-1169.

4. Carvalho TS, Perez LR. Impact of biofilm production on polymyxin B susceptibility among Pseudomonas aeruginosa clinical isolates. Infect Control Hosp Epidemiol 2019. DOI: 10.1017/ice.2019.85.

5. Naparstek L, Carmeli Y, Navon-Venezia S, Banin E. Biofilm formation and susceptibility to gentamicin and colistin of extremely drug-resistant KPC-producing Klebsiella pneumoniae. J Antimicrob Chemother 2014;69:1027-1034.
6. Lee MY, Ko KS, Song JH, Peck KR. In vitro effectiveness of the antibiotic lock technique (ALT) for the treatment of catheter-related infections by Pseudomonas aeruginosa and Klebsiella pneumoniae. J Antimicrob Chemother 2007;60:782-787.

7. Perez LRR. Why susceptible bacteria may become resistant to infection control measures? A Pseudomonas biofilm example. Infect Control Hosp Epidemiol 2019;40:386-388.

8. Stewart PS. Mechanisms of antibiotic resistance in bacterial biofilms. Int $J$ Med Microbiol 2002;292:107-113.

9. Andrade LN, Curiao T, Ferreira JC, et al. Dissemination of $b l a_{\mathrm{KPC}-2}$ by the spread of Klebsiella pneumoniae clonal complex 258 clones (ST258, ST11, ST437) and plasmids (IncFII, IncN, IncL/M) among Enterobacteriaceae species in Brazil. Antimicrob Agents Chemother 2011;55:3579-3583.

\section{Prevalence of bloodstream infections in neutropenic patients with bacteriuria}

\section{Erica S. Herc MD ${ }^{1,2}$ (1), Rachelle N. Rivera MD³ , Dale Bixby MD ${ }^{4}$ and Carol E. Chenoweth MD ${ }^{5}$}

${ }^{1}$ Division of Infectious Diseases, Department of Internal Medicine, Henry Ford Hospital, Detroit, Michigan, ${ }^{2}$ Wayne State University School of Medicine, Detroit, Michigan, ${ }^{3}$ Renown Health, Reno, Nevada, ${ }^{4}$ Division of Hematology and Oncology, Department of Internal Medicine, Michigan Medicine, Ann Arbor, Michigan and ${ }^{5}$ Division of Infectious Diseases, Department of Internal Medicine, Michigan Medicine, Ann Arbor, Michigan

To the Editor-Patients with hematologic malignancies and neutropenia are at increased risk for several infectious complications. They are predisposed to higher rates of bloodstream infections (BSIs) than the general population. Although BSI is caused by bacteriuria in $\sim 3 \%$ of cases within the general population, ${ }^{1}$ malignancy (odds ratio [OR], 1.94) and receiving immunosuppression within 14 days of bacteriuria $(\mathrm{OR}, 8.13)$ are risk factors for urinary-tract-

Author for correspondence: Erica Herc, Email: eherc1@hfhs.org

Cite this article: Herc ES, et al. (2019). Prevalence of bloodstream infections in neutropenic patients with bacteriuria. Infection Control \& Hospital Epidemiology, 40: 955-956, https://doi.org/10.1017/ice.2019.139 related BSIs. ${ }^{2}$ However, few studies have evaluated the occurrence of secondary BSIs from bacteriuria in neutropenic patients. This study was performed to define the incidence of secondary BSIs in patients with acute myeloid leukemia (AML) with neutropenia and bacteriuria and to determine whether neutropenic patients with asymptomatic bacteriuria have a higher rate of BSI than the general population.

Our study took place in a 900-bed Midwestern tertiary-care hospital with $\sim 2,000$ annual hematology and oncology admissions. We performed a cohort study of all adult patients with AML who were admitted to the hematology service between February 2010 and November 2013. A case was defined as any patient with neutropenia and bacteriuria during the study period. 